American Journal of Infectious Diseases 4 (4): 267-271, 2008

ISSN 1553-6203

(C) 2008 Science Publications

\title{
The Effect of a 4th Generation-Cephalosporin Introduction upon the Incidence of Multidrug-Resistant Gram-Negative Bacteria in a Non-Teaching Hospital
}

\author{
${ }^{1,2}$ Guilherme Henrique Campos Furtado, ${ }^{1,2}$ Luciana Baria Perdiz and \\ ${ }^{1}$ Eduardo Alexandrino Servolo Medeiros \\ ${ }^{1}$ Hospital Epidemiology Committee, Hospital São Paulo, Division of Infectious Diseases, \\ Department of Medicine, Federal University of São Paulo, Brazil \\ ${ }^{2}$ Infection Control Committee, Hospital Estadual de Diadema, Diadema, Brazil
}

\begin{abstract}
Problem statement: Antimicrobial resistance is a worrisome situation in hospitals around the world and the misuse of certain classes of antimicrobials has contributed for this situation. Approach: We performed a prospective surveillance study on the incidence of multi-drug resistant bacteria before (phase 1) and after (phase 2) the introduction of a 4th-generation cephalosporin in a non-teaching hospital. Results: There was a significant reduction in the incidence of Enterobacter species (from 1.3 isolates per 100 patient-days to 0.39 isolates per 100 patient-days, $p=0.01$ ) between the two periods. We also observed a reduction in the consumption of ceftazidime (from 64.3 DDD per 1000 patient-days to 29.6 DDD per 1000 patient-days, $p=0.002$ ) and ceftriaxone (from 323.9 DDD per 1000 patient-days to 246.2 DDD per 1000 patient-days, $\mathrm{p}=0.01$ ). Conclusion: The introduction of a 4th-generation cephalosporin in our setting resulted in an important reduction in the incidence of Enterobacter species.
\end{abstract}

Key words: $4^{\text {th }}$ generation-cephalosporin,incidence,multidrug-resistant,Gram-negative bacteria

\section{INTRODUCTION}

Background: In the last two decades, antimicrobial resistance has become one of the greatest health problems, mainly in hospitals. Despite the continuous development and introduction of new antibiotics, resistance continues to increase progressively in several microorganisms. Over $70 \%$ of the bacteria found in the United States hospitals are resistant to at least one antibiotic $^{[1]}$. Nosocomial infections caused by these pathogens are an important cause of morbidity and mortality, especially in intensive care units. The major microorganisms involved are: Gram-positive (methicillin-resistant Staphylococcus aureus and vancomycin-resistant Enterococcus) and Gramnegative, such as carbapenem-resistant Pseudomonas aeruginosa and Acinetobacter spp., Extended -Spectrum Beta-Lactamase (ESBL) producing Klebsiella pneumoniae and E. coli and ampC-producing Enterobacter spp.

There are several factors contributing to the increased antimicrobial resistance. Some are related to characteristics of the host; for example: Patients with severe disease, immunocompromised patients, new invasive procedures and lack of compliance with infection control measures ${ }^{[2]}$. Other factors are related to the selective pressure from exaggerated use of antimicrobials. McGowan ${ }^{[3]}$ warned two decades ago that the use of antimicrobials is one of the most important factors in the development of resistance and this problem continues to be very relevant in the present day.

The use of certain classes of antimicrobials, such as 3rd-generation cephalosporins is implicated in the development of resistance caused by enzymes in the group of

ESBLs $^{[4-6]}$. Restriction of such drugs causes a reduction in the incidence of these beta-lactamases in hospitals $^{[4,6]}$.

Due to its singular properties, 4th-generation cephalosporins appeared in recent years as an important option to 3rd-generation cephalosporins, mainly due to its enhanced activity against Enterobacter spp. and other ampC-beta-lactamase producers (e.g., Citrobacter, Serratia, Proteus) and lower selection of ESBLs. The cyclical introduction and withdrawal of antimicrobial agents have been indicated as one of the possible methods of controlling antimicrobial resistance, especially in intensive care units ${ }^{[7]}$.

Corresponding Author: Eduardo Alexandrino Servolo de Medeiros, Rua Napoleão de Barros, 690, 2 Andar, Zip Code: 04024-002, São Paulo, Brazil Ph/Fax: 55-11- 5571-8935 
The goal of this prospective study was to evaluate the effect of the introduction of an 4th-generation cephalosporin on incidence of multi-drug resistant gram-negative pathogens.

\section{MATERIALS AND METHODS}

Setting: The Diadema state hospital is a public community hospital with 180 beds, located in the city of Diadema in the metropolitan region of São Paulo. It holds three intensive care units (medical-surgical, pediatric and neonatal) and medical and surgical wards including orthopedics, gastrosurgery, neurosurgery and thoracic surgery. There are no transplant, oncology and hematology wards.

Study protocol: A one-year prospective observational study was conducted in order to observe alterations in the incidence of major pathogens isolated from clinical cultures, as well as changes in the quantity of antimicrobials used after the introduction of cefepime, a 4th generation cephalosporin.

The study was divided into two six-month periods. The first period from June-December 2002 represented the period prior to using cefepime in the hospital. The second period, from December 2002-May 2003, when this antibiotic was introduced as a therapeutic option for nosocomial pathogens.

The Infection control committee was comprised by an infection control physician and an epidemiologist nurse. The control of antimicrobial medications was done through an order form filled out by the assistant doctors and had already been in use for the last eighteen months. The order form was then evaluated by the Infection Control Committee daily or within $72 \mathrm{~h}$ on weekends. The record keeping was obligatory for the following antimicrobials: 3rd or 4th generation cephalosporins, ciprofloxacin, vancomycin, carbapenens, metronidazole, clindamycin and polymyxin B. The evaluation of the order form included indication, dosage and timing of use and had a validity of seven days that could be renewed.

There was no systematic replacement of any antimicrobial or class by cefepime. However, assistant physicians were informed about the risks for the selection of bacterial resistance associated with the overuse of the third-generation cephalosporins, as well as the benefits of their replacement with cefepime.

There was no evaluation if the positive cultures were associated with infection or colonization.

The use of antibiotics was provided in Defined Daily Doses (DDD) per 1000 patient-days, in accordance with the World Health Organization recommendation ${ }^{[8]}$. Five antimicrobials were selected for the comparative evaluation of the two study periods: ceftazidime, ceftriaxone, ciprofloxacin, meropenem and imipenem. The choice of drugs was made by their importance in the treatment of nosocomial infections caused by gram-negative pathogens. To evaluate the impact of the introduction of a forth-generation cephalosporin on the incidence of multi-drug resistant pathogens, the following multi-drug resistant species were selected: Carbapenem-resistant Pseudomonas aeruginosa and Acinetobacter baumannii, Extended-Spectrum-Beta-Lactamase producing Klebsiella pneumoniae and Escherichia coli, Enterobacter spp. resistant to 3rd-generation cephalosporin.

The cultures were carried out at the hospital's microbiology laboratory by diffusion disk technique, standardized by NCCLS ${ }^{[9]}$. No MIC test was performed.

Statistical analysis: Rates were analyzed using chisquare test or Fischer's exact test. All p values are two-sided. All interval estimates are $95 \%$ confidence intervals. SPSS program for Windows (version 10.0; SPSS, Inc., Chicago, IL) was used.

\section{RESULTS}

We analyzed 243 cultures in 159 patients and 195 cultures in 148 patients in the first and second period respectively. The mean number of admissions per month and patient-days per month were 889 and 4372 respectively during the study period.

There were an average 30.4 and $23.6 \%$ of multidrug resistant pathogens in the first and second period respectively. Comparative data concerning the incidence of multi-drug resistant microorganisms are provided in Table 1 . There was a $6.8 \%$ reduction in the number of positive cultures of multidrug-resistant gram-negative bacteria between the two periods. The most expressive reduction was verified in Enterobacter spp. resistant to 3rd generation cephalosporin (from 1.3 isolates per 100 patient-days to 0.3 isolates per 100 patient-days, $\mathrm{p}<0.01)$. Extended-Spectrum-Beta-Lactamase producing Klebsiella pneumoniae, Extended-Spectrum-BetaLactamase producing E. coli, carbapenem-resistant Pseudomonas aeruginosa and Acinetobacter baumannii had non-significant decrease in their incidence between the two periods.

There was a significant reduction in the consumption of ceftazidime (from 64.3 DDD per 
1000 patient-days to 29.6 DDD per 1000 patientdays, $\mathrm{p}<0.05$ ) and ceftriaxone (from 323.9 DDD per 1000 patient-days to 246.2 DDD per 1000 patient-days, $\mathrm{p}<0.05)$. Table 2 shows the consumption of the antimicrobial drugs in Defined Daily Dose (DDD) per 1000 patient-days. Urine was the major source of isolation during the two periods. Table 3 shows the cultures per site of isolation. Medical ward followed by Adult ICU were the units with the highest number of isolates during the two periods. Table 4 shows the number of cultures per unit of isolation.

Table 1: Number of cultures of multidrug-resistant pathogens per 100 patient-days

\begin{tabular}{llll}
\hline $\begin{array}{l}\text { Organism/phenotypic } \\
\text { resistance }\end{array}$ & 1st period & 2nd period & $\mathrm{P}^{\mathrm{a}}$ \\
\hline $\begin{array}{l}\text { ESBL-producing } \\
\text { Klebsiella pneumoniae }\end{array}$ & 1.4 & 1.18 & 0.48 \\
$\begin{array}{l}\text { 3rd gen. Ceph-resistant } \\
\text { Enterobacter } \text { spp. }\end{array}$ & 1.3 & 0.39 & 0.01 \\
$\begin{array}{l}\text { ESBL-producing } \text { E. coli } \\
\begin{array}{l}\text { Imipenem-resistant } \\
P . \text { aeruginosa }\end{array}\end{array}$ & 0.38 & 0.11 & 0.57 \\
$\begin{array}{l}\text { Imipenem-resistant } \\
\text { Acinetobacter baumannii }\end{array}$ & 1.37 & 0.78 & 0.09 \\
& 0.04 & 0.02 & 1.00
\end{tabular}

Acinetobacter baumannii

ESBL: Extended Spectrum Beta Lactamase; 3rd gen: Ceph third generation cephalosporin; ${ }^{\text {a }}$ : Determined by chi-square test

Table 2: Antimicrobial drugs in Defined Daily Dose (DDD) per 1000 patients-days

\begin{tabular}{lrrl}
\hline Antimicrobials & 1st period & 2nd period & $\mathrm{P}^{\mathrm{a}}$ \\
\hline Ceftazidime & 64.35 & 29.67 & 0.002 \\
Ceftriaxone & 323.96 & 246.21 & 0.010 \\
Cefepime & 0.00 & 21.92 & ----- \\
Meropenem & 16.41 & 17.38 & 0.690 \\
Imipenem & 29.25 & 25.10 & 0.480 \\
Ciprofloxacin & 19.22 & 22.90 & 0.240 \\
\hline
\end{tabular}

${ }^{\text {a }}$ : Determined by chi-square test

Table 3: Number of cultures per site of isolation

\begin{tabular}{lll}
\hline Site & 1st period N $(\%)$ & 2nd period N $(\%)$ \\
\hline Urine & $91(38.0 \%)$ & $81(41.7 \%)$ \\
Blood & $50(20.9 \%)$ & $28(14.4 \%)$ \\
Respiratory secretions & $35(14.6 \%)$ & $38(19.5 \%)$ \\
Other secretions & $30(12.5 \%)$ & $25(12.8 \%)$ \\
Catheter tip & $13(5.4 \%)$ & $10(5.1 \%)$ \\
Other & $29(8.3 \%)$ & $11(6.1 \%)$ \\
\hline
\end{tabular}

Table 4: Number of cultures per unit of isolation

\begin{tabular}{lll}
\hline Site & 1st period N $(\%)$ & 2nd period N $(\%)$ \\
\hline Medical ward & $121(48.7 \%)$ & $81(48.5 \%)$ \\
Adult ICU & $45(18.1 \%)$ & $35(20.9 \%)$ \\
Pediatric ward & $28(11.2 \%)$ & $17(10.1 \%)$ \\
Surgical ward & $22(8.8 \%)$ & $16(9.5 \%)$ \\
Neonatal ICU & $19(7.6 \%)$ & $13(7.7 \%)$ \\
Pediatric ICU & $13(5.2 \%)$ & $5(2.9 \%)$ \\
\hline ICU: In
\end{tabular}

ICU: Intensive Care Unit

\section{DISCUSSION}

Antimicrobial resistance is an important source of concern in the last two decades. Prevention of the emergence of resistance and the dissemination of multidrug resistant pathogens result in a decrease in the morbidity, mortality and costs of health care ${ }^{[7]}$. The excessive and inappropriate use of antimicrobials is the main cause underlying the emergence of resistance and continues to be a problem despite the existence of published guidelines and the implementation of antimicrobial restriction policies in many hospitals ${ }^{[10-13]}$. Several strategies for regulating antimicrobial prescribing practices have been proposed, such as: formulary replacement or restriction ${ }^{[14]}$; introduction of order forms ${ }^{[15]}$; education programs for health care providers; feedback activities ${ }^{[16]}$; and approval required from the infectious diseases physician for drug prescription ${ }^{[17]}$.

There are two major approaches to reducing excessive or inappropriate use of antimicrobials: those that attempt to optimize usage and those that tend to optimize the availability of specific agents ${ }^{[18]}$.

Changing the availability of specific agents is very attractive to hospitals in the view of the simplicity in implementation ${ }^{[18]}$. We introduced a forth-generation cephalosporin in our hospital as a new antimicrobial option to treat nosocomial infections, after which we observed changes in the incidence of multi-drug resistant microorganisms. We also observed alterations in the consumption of the major antimicrobial drugs utilized for treating infections caused by Gram-negative pathogens.

We observed a statistically significant reduction in the incidence of Enterobacter spp. isolates confirming the good activity of forth-generation cephalosporins against this pathogen.

When only a single antimicrobial change is performed this is often referred to as a "single switch" in that the physicians change the prescription from one specific agent or class of agents to another agent ${ }^{[18]}$. Kollef et al. ${ }^{[19]}$, switched the antimicrobial agent of choice for empirical therapy for suspected gramnegative bacterial infection in the cardiothoracic ICU from ceftazidime to ciprofloxacin. The percentage of patients who were infected with ceftazidime-resistant gram-negative bacteria was reduced by $25 \%$.

The introduction of cefepime also resulted in an expressive reduction in the consumption of ceftriaxone and ceftazidime. Probably there was a switch from these to cefepime. There was also a reduction in the use of imipenem, probably because cefepime was used as empirical therapy in nosocomial infections replacing 
imipenem in certain situations. The reduction in the use of 3rd-generation cephalosporin may have contributed to a lower incidence of ESBL-producing Klebsiella pneumoniae and E. coli. There is almost a universal agreement that this type of antimicrobial resistance is related to selective pressure exerted by the use of these agents ${ }^{[20,21]}$. Fourth-generation cephalosporins pose no beneficial activity against this kind of resistance and their introduction alone probably would not have an impact on these pathogens.

\section{CONCLUSION}

In summary, our study demonstrated the impact of the introduction of a forth-generation-cephalosporin on the incidence of multi-drug resistant gram-negative pathogens. Nonetheless, it is important to emphasize the short period of observation and the probability that new kinds of resistance will emerge with this recent antimicrobial drug introduction.

\section{REFERENCES}

1. Bruning, L.M., 1996. Emerging infectious diseases: Threats to the OR? Today's Surg. Nurse, 18: 21-23.

2. Murthy, R., 2001. Implementation of strategies to control antimicrobial resistance. Chest, 119: 405S-411S. DOI: 10.1378/chest.119.2_suppl.405S

3. McGowan, J.E., 1983. Antimicrobial organisms and its relation to antibiotic use. Rev. Infect. Dis., 5: 1033-1048. http://www.ncbi.nlm.nih.gov/pubmed/6318289

4. Meyer, K.S., C. Urban and J.A. Eagan et al., 1993. Nosocomial outbreak of Klebsiella infection resistant to late-generation cephalosporins. Ann. Intern. $\quad$ Med., 119: 353-358. http://www.annals.org/cgi/content/full/119/5/353

5. Patterson, J.E., T.C. Hardin and C.A. Kelly et al., 2000. Association of antibiotic utilization measures and control of multiple-drug resistance in Klebsiella pneumoniae. Infect. Control Hosp. Epidemiol., 21: 455-458. http://www.ncbi.nlm.nih.gov/pubmed/10926395

6. Rahal, J.J., C. Urban and D. Horn et al., 1998. Class restriction of cephalosporin use to control total cephalosporin resistance in nosocomial Klebsiella. J. Am. Med. Assoc., 280: 1233-1237. http://www.ncbi.nlm.nih.gov/pubmed/9786372

7. Shlaes, D.M., D.N. Gerding and J.F. John Jr. et al., 1997. Society for healthcare epidemiology of america and idsa joint committee on the prevention of antimicrobial resistance: Guidelines for the prevention of antimicrobial resistance in hospitals. Clin. Infect. Dis., 25: 584-599. DOI: 10.1086/513766
8. Maxwell, M., D. Heaney, J.G. Howie and S. Noble, 1993. General practice fund holding observations on prescribing patterns and costs using the defined daily dose method. Br. Med. J., 307: 1190-1194. http://www.pubmedcentral.nih.gov/articlerender.fc gi?artid=1679327

9. National Committee for Clinical Laboratory Standards, 2002. Performance Standards for Antimicrobial Disk Susceptibility Testing. 12th Informational Supplement. Document M100-S12. NCCLS, Wayne, PA.

10. Dellinger, E.P., P.A. Gross and T.L. Barrett et al., 1994. Quality standard for antimicrobial prophylaxis in surgical procedures. Clin. Infect. Dis., 18: 422-427.

http://md1.csa.com/partners/viewrecord.php?reque ster $=$ gs \&collection $=$ ENV\&recid $=3709409 \& q=\&$ ui $\mathrm{d}=\&$ setcookie $=$ yes

11. Lomas, J., G.M. Anderson, K. Dormick-Pierre, E. Vayda, M.W. Enkin and W.J. Hannah, 1989. Do practice guidelines guide practice? The effect of a consensus statement on the practice of Physicians. N. Engl. J. Med. 321: 1306-1311. http://content.nejm.org/cgi/content/abstract/321/19/ 1306

12. Nickman, N.A., H.F. Blissenbach, J.D. Herrick, 1984. Medical committee enforcement of a policy limiting postsurgical antibiotic use. Am. J. Hosp. Pharm., 41: 2053-2056.

http://www.ajhp.org/cgi/content/abstract/41/10/2053

13. Woodward, R.S., G. Medoff, M.D. Smith and J.L. Gray, 1987. III Antibiotic cost savings from formulary restrictions and physician monitoring in a medical-school-affiliated hospital. Am. J. Med., 83: 817-23.

http://www.ncbi.nlm.nih.gov/pubmed/3674089

14. Lee, J., J.A. Carlson, M.A. Chamberlain, 1995. A team approach to hospital formulary replacement. Diagn. Microbiol. Infect. Dis., 22: 239-242. http://cat.inist.fr/?aModele=afficheN\&cpsidt=3626534

15. Gyssens, I.C., W.L. Blok, P.J. Van den Broek, Y.A. Hekster and J.W. van der Meer, 1997. Implementation of an educational program and an antibiotic order form to optimize quality of antimicrobial drug use in a department of internal medicine. Eur. J. Clin. Microbiol. Infect. Dis., 16: 904-912. DOI: 10.1007/BF01700557

16. De Santis, G., K.J. Harvey, D. Howard, M.L. Mashford and R.F. Moulds, 1994. Improving the quality of antibiotic prescription patterns in general practice: the role of educational intervention. Med. J. Aust., 160: 502-505. http://www.ncbi.nlm.nih.gov/pubmed/8170427 
17. Landman, D., M. Chockalingam and J.M. Quale, 1999. Reduction in the incidence of methicillinresistant Staphylococcus aureus and ceftazidimeresistant Klebsiella pneumoniae following changes in a hospital antibiotic formulary. Clin. Infect. Dis., 28: 1062-1066. DOI: 10.1086/514743

18. Fridkin, S.K., 2003. Routine cycling of antimicrobial agents as an infection-control measure. Clin. Infect. Dis., 36: 1438-1444. DOI: $10.1086 / 375082$

19. Kollef, M.H., J. Vlasnik, L. Sharpless, C. Pasque, D. Murphy and V. Fraser, 1997. Scheduled change of antibiotic classes: A strategy to decrease the incidence of ventilator-associated pneumonia. Am. J. Respir. Crit. Care Med., 156: 1040-1048. http://ajrccm.atsjournals.org/cgi/content/abstract/15 $6 / 4 / 1040$
20. Duncan, R.A., 1997. Controlling use of antimicrobial agents. Infect. Control Hosp. Epidemiol., $\quad$ 18: 260-266. http://www.ncbi.nlm.nih.gov/pubmed/9131372

21. Tenover, F.C. and J.M. Hughes, 1996. The challenge of emerging infectious diseases: development and spread of multiply-resistant bacterial pathogens. J. Am. Med. Assoc., 275: 300-304. http://jama.amaassn.org/cgi/content/abstract/275/4/300 